\title{
The significance of multi-line chemotherapy for advanced gastric cancer: A retrospective analysis
}

\author{
MEIQIN YUAN ${ }^{1}$, ZHONG SHI $^{1}$, ZENG WANG ${ }^{2}$, WANGXIA LV ${ }^{1}$, \\ YUNSHAN YANG ${ }^{1}$, FANGXIAO LU ${ }^{3}$, YAZHEN ZHAO ${ }^{1}$ and HAIJUN ZHONG ${ }^{1}$ \\ Departments of ${ }^{1}$ Chemotherapy, ${ }^{2}$ Pharmacy and ${ }^{3}$ Radiology, \\ Zhejiang Cancer Hospital, Hangzhou, Zhejiang 310022, P.R. China
}

Received July 4, 2016; Accepted December 22, 2016

DOI: $10.3892 / \mathrm{mco} .2017 .1163$

\begin{abstract}
Palliative chemotherapy is known to benefit patients with advanced gastric cancer by palliating symptoms and improving survival. The aim of the present study was to evaluate the efficacy and toxicity of chemotherapy regimens that are commonly used in patients with advanced or recurrent gastric cancer. Patients with advanced or recurrent gastric cancer who were treated by at least two chemotherapy regimens between May 2006 and July 2014 at Zhejiang Cancer Hospital (Hangzhou, China) were retrospectively investigated. Survival was evaluated using the Kaplan-Meier method. A total of 248 patients were reviewed, and 158 were evaluated in the final analysis, with a median age of 57 years and a Karnofsky performance status score of $\geq 80$. The median progression-free survival (PFS) time was 168 days for first-line chemotherapy, 96 days for second-line chemotherapy, and the median overall survival (OS) time was 356 days. Further analysis revealed that patients with the disease controlled [complete response $(\mathrm{CR})+$ partial response $(\mathrm{PR})+$ stable disease $(\mathrm{SD})]$, no matter whether they received first-or second-line chemotherapy, may have had an improved OS compared with patients with disease progression (PD). Patients who were treated with $>2$ lines of chemotherapy had an improved OS compared those who ceased treatment following failure of the second-line chemotherapy. The cycle number of chemotherapy that patients received was associated with OS. The site of the primary and metastatic tumors was also associated with OS. Other factors, including gender, age, histological type, whether a radical operation was received, and chemotherapy regimens, had no evident association with survival. The toxicities were generally tolerated. Taken together, the results from the present study have demonstrated that an increased cycle number of
\end{abstract}

Correspondence to: Dr Haijun Zhong, Department of Chemotherapy, Zhejiang Cancer Hospital, 38 Guangji Road, Hangzhou, Zhejiang 310022, P.R. China

E-mail: zhj1712@aliyun.com

Key words: advanced gastric cancer, palliative chemotherapy, retrospective analysis, progression-free survival, overall survival effective chemotherapy may prolong the survival of patients with advanced gastric cancer. Differences among the chemotherapy regimens had no clear correlation with survival.

\section{Introduction}

Gastric cancer is the fifth most common malignancy, and has the third highest rate of cancer-specific mortality worldwide (1). In excess of $70 \%$ of the cases worldwide occur in developing countries, and over half occur in Eastern Asia, predominantly in China (1). A large number of patients are diagnosed at an inoperable stage of the disease, or experience disease recurrence, indicating a poor outcome (2). The 5-year survival rate of advanced gastric cancer (AGC) was $<1 \%$, and the median overall survival (OS) rate was determined to be $<1$ year (3). Palliative chemotherapy is an important treatment option for patients with AGC, since it improves survival and the quality of life in patients with a good performance status (4).

Systemic chemotherapy, with a combination of fluoropyrimidine and platinum, is now regarded as the standard treatment for these patients (4). Over the course of the last few years, several single or combined chemotherapy regimens containing S-1, capecitabine, platinum agents (cisplatin and oxaliplatin), taxanes (paclitaxel and docetaxel) and irinotecan have demonstrated potent effects for gastric cancer (5-11). In addition, a trastuzumab for gastric cancer (ToGA) study reported a clinical benefit of trastuzumab treatment for patients with human epidermal growth factor receptor 2 (HER2)-positive gastric cancer (12).

However, the efficacy of first-line treatment is modest, and the majority of patients either do not respond to therapy, or eventually experience disease progression. Patients with refractory gastric cancer often receive salvage chemotherapy clinically. The results of two randomized controlled trials (RCTs) were published, revealing an OS benefit from treatments with irinotecan or docetaxel compared with best supportive care (BSC) alone in patients for whom one or two prior treatments had failed (13,14). Recently, Kim et al (15) reported a meta-analysis in which a significant reduction in the risk of death was observed in patients receiving salvage chemotherapy compared with supportive cancer treatment. This provided evidence for implementing second-line chemotherapy in patients with AGC. Based on the variety 
of patients, making the strategy for optimizing the regimens and arranging the order of different regimens for individual patients occasionally remain difficult for oncologists.

In the present study, the efficacy and safety of first- and second-line chemotherapy in AGC patients was evaluated with a retrospective analysis, the aim being to investigate the best strategy for choosing chemotherapy regimens and to identify whether increasing the number of treatment cycles will benefit patients.

\section{Patients and methods}

Patients. Patients with advanced or recurrent gastric cancer, who were treated with at least two chemotherapy regimens between May 2006 and July 2014 at Zhejiang Cancer Hospital (Hangzhou, China), were retrospectively investigated. Patients with histologically confirmed advanced or recurrent gastric cancer were retrospectively investigated. Chemotherapy regimens were unrestricted. Furthermore, the eligibility criteria included at least one measurable lesion of $\geq 1 \mathrm{~cm}$ in the longest diameter, or a lymphonodus of $\geq 1.5 \mathrm{~cm}$ in the shortest diameter. The present study was approved by the Ethics Committee and Institutional Review Board of Zhejiang Cancer Hospital (Hangzhou, China), and was conducted in compliance with the Declaration of Helsinki. Patients were followed up until death, or until December 31, 2014 if the patients were alive at that time.

Treatments. Chemotherapy regimens were unrestricted in the present study. Systemic chemotherapy was either mono- or combination chemotherapy, which demonstrated efficacy in gastric cancer, including S1, capecitabine $\left(\right.$ Xeloda $\left.^{\circledR}\right)$, CPT-11 (irinotecan), SP, S-1 plus oxaliplatin (SOX), capecitabine plus oxaliplatin (XELOX), capecitabine plus docetaxel (XT), folinic acid, fluorouracil and oxaliplatin (FOLFOX), folinic acid, fluorouracil and irinotecan (FOLFIRI), docetaxel and cisplatin (DP), docetaxel and S-1 (DS), docetaxel plus capecitabine (DX), docetaxel, cisplatin and 5-fluorouracil DCF, and so forth. The doses of chemotherapy, and their adjustments, were determined according to the specific situation of each patient. After progression of the disease had occurred, it usually became necessary to switch to another chemotherapy regimen, or to stop chemotherapy and continue with the best support treatment.

Adverse effects. Toxicity was measured using the National Cancer Institute-Common Toxicity Criteria, version 2.0 toxicity scales (16). Grade 3 to 4 toxicity was recorded according to the medical records.

Assessment and statistics. The response was evaluated every two cycles of treatment using the Response Evaluation Criteria in Solid Tumors (17). Survival time was analyzed using the Kaplan-Meier software of SPSS, version 15.0 (SPSS, Inc., Chicago, IL, USA). $\mathrm{P}<0.05$ was considered to indicate a statistically significant value.

\section{Results}

Patient characteristics and clinical data. A total of 248 patients with advanced or recurrent gastric cancer who had received at least two chemotherapy regimens were reviewed. Of those patients, 90 were excluded due to incomplete follow-up data and an inability to perform a therapeutic efficacy evaluation. This resulted in a total of 158 patients who were suitable for a final evaluation.

The median age of the patients was 57 years and the Karnofsky Performance Status (KPS) score was $\geq 80$. A detailed description of patient characteristics is shown in Table I. Treatment conditions of the patients are shown in Table II, and the specific regimens used in first- and second-line chemotherapies are shown in Table III.

Survival analysis. The Kaplan-Meier distribution curves of progression-free survival (PFS) are shown in Fig. 1A and B for first- and second-line chemotherapy, respectively. The PFS was 168 days [95\% confidence interval (CI), 140.6-195.4 days] in first-line chemotherapy, and 96 days (95\% CI, 84.0-108.0 days) in second-line chemotherapy.

The Kaplan-Meier distribution of OS is shown in Fig. 1C. The median OS was 356 days (95\% CI, 323.7-388.4 days). Further subgroup analyses revealed that OS was significantly longer for the following groups: Esophagogastric junction tumor site $(\mathrm{P}=0.016$; Fig. 2A) and lymphatic metastasis $(\mathrm{P}=0.005$; Fig. 2B). The histological factor appeared to correlate with OS, of which patients with well-differentiated tumors had the best OS, patients with poorly differentiated tumors performed more poorly, and patients of 'differentiation unknown' status had the worst OS; however, the difference was not significant $(\mathrm{P}=0.687)$. Other factors, including gender, age, and whether a radical operation was received, had no clear correlation with survival (Table I). Several of the treatment conditions were significantly associated with longer OS, as follows: Treated with $>2$ lines of chemotherapy $(\mathrm{P}<0.001$; Fig. 2C), increased number of cycles of first-(P<0.001; Fig. 2D) and second-( $\mathrm{P}<0.001$; Fig. $2 \mathrm{E})$ line of chemotherapy, and good short-term efficacy of first- $(\mathrm{P}<0.001$; Fig. $2 \mathrm{~F})$ and second$(\mathrm{P}<0.001$; Fig. 2G) line of chemotherapy. Treatment with more drugs in combination chemotherapy resulted in longer OS, although the difference was not significant. Differences between chemotherapy regimens had no obvious correlation with survival.

Safety. According to medical records, the toxicities were, in the main, well tolerated. Grade 3 or 4 adverse events included neutropenia $(0.6 \%$ in the first-line chemotherapy in a triple regimen, and $0.6 \%$ in the second-line chemotherapy) and gastrointestinal reactions $(0.6 \%)$. In addition, one patient developed grade 2 weight loss on receiving second-line chemotherapy. No neutropenic fever or treatment-associated mortalities were documented.

\section{Discussion}

Compared with best supportive care (BSC), chemotherapy is more beneficial for patients with AGC in terms of being able to alleviate symptoms and prolong survival $(18,19)$. Based on these published studies, the median PFS was 4-6 months, and the median OS was 7-13 months for patients who received chemotherapy. In the present study, similar results were obtained: The median PFS was 168 days in first-line chemotherapy, 96 days in second-line chemotherapy, and the median 
Table I. Baseline characteristics of patients and related overall survival (OS) ( $n=158)$.

\begin{tabular}{|c|c|c|c|}
\hline Character & $\mathrm{n}(\%)$ & mOS (days) & P-value \\
\hline Gender & & & 0.469 \\
\hline Male & $104(65.8)$ & 335 & \\
\hline Female & $54(34.2)$ & 375 & \\
\hline Age & & & - \\
\hline Median & 57 & - & \\
\hline Range & $24-76$ & & \\
\hline$\leq 60$ years & $63(39.9)$ & 351 & 0.793 \\
\hline$>60$ years & $95(60.1)$ & 364 & \\
\hline Radical operation & & & 0.980 \\
\hline Yes & $44(27.8)$ & 333 & \\
\hline No & $114(72.2)$ & 358 & \\
\hline Pathology & & & 0.687 \\
\hline Well differentiated & $1(0.6)$ & 545 & \\
\hline Moderately differentiated & $17(10.8)$ & 372 & \\
\hline Poorly differentiated & $114(72.2)$ & 358 & \\
\hline Differentiation unknown & $26(16.4)$ & 320 & \\
\hline Primary tumor site & & & 0.016 \\
\hline Esophagogastric junction & $23(14.6)$ & 464 & \\
\hline Body of stomach & $68(43.0)$ & 329 & \\
\hline Gastric antrum & $45(28.5)$ & 405 & \\
\hline Diffuse gastric lesions & $18(11.4)$ & 356 & \\
\hline Unkown & $4(2.5)$ & 251 & \\
\hline Metastasis sites & & & 0.005 \\
\hline Lymphatic metastasis & $38(24.0)$ & 412 & \\
\hline Hematogenous metastasis & $26(16.5)$ & 355 & \\
\hline Peritoneal metastasis & $30(19.0)$ & 392 & \\
\hline Mixture metastasis & $64(40.5)$ & 304 & \\
\hline
\end{tabular}

mOS, median overall survival.
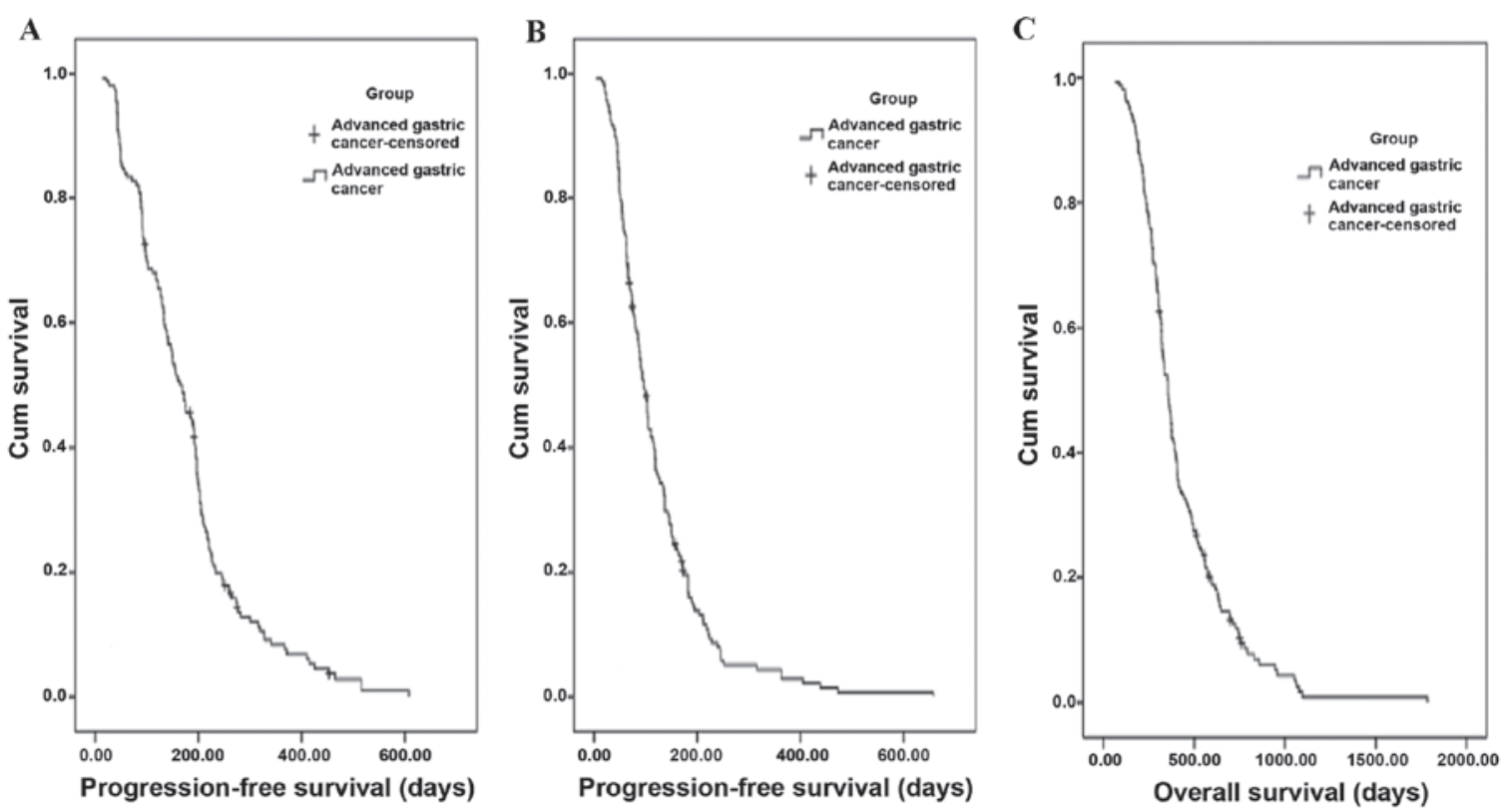

Figure 1. Kaplan Meier distribution curves of PFS (days) and OS (days) of all the patients. (A) PFS of first-line chemotherapy; the median PFS was 168 days; (B) PFS of second-line chemotherapy; the median PFS was 96 days. (C) OS; the median OS was 356 days. OS, overall survival; PFS, progression-free survival. 
Table II. Treatment conditions of patients and related overall survival (OS) ( $n=158)$.

\begin{tabular}{|c|c|c|c|}
\hline Character & $\mathrm{n}(\%)$ & mOS (days) & P-value \\
\hline Lines of chemotherapy & & & $<0.001$ \\
\hline$\leq 2$ & $103(65.2)$ & 316 & \\
\hline$>2$ & $55(34.8)$ & 487 & \\
\hline First-line chemotherapy & & & 0.647 \\
\hline \multicolumn{4}{|l|}{ Regimen } \\
\hline Monochemotherapy & $9(5.7)$ & 364 & \\
\hline Doublet chemotherapy & $134(84.8)$ & 356 & \\
\hline Triple chemotherapy & $15(9.5)$ & 321 & \\
\hline Regimen containing oxaliplatin & $76(48.1)$ & 356 & 0.548 \\
\hline Regimen containing taxanes & $41(25.9)$ & 314 & \\
\hline Regimen containing Irinotecan and others & $41(25.9)$ & 392 & \\
\hline Cycles (mean, 5; range, 1-12) & & & $<0.001$ \\
\hline $1-2$ cycles & $28(17.7)$ & 219 & \\
\hline $3-4$ cycles & $38(24.0)$ & 327 & \\
\hline $5-6$ cycles & $60(38.0)$ & 405 & \\
\hline$>6$ cycles & $32(20.3)$ & 403 & \\
\hline Short-term efficacy & & & $<0.001$ \\
\hline $\mathrm{CR}+\mathrm{PR}+\mathrm{SD}$ & $112(70.9)$ & 403 & \\
\hline PD & $46(29.1)$ & 228 & \\
\hline Second-line chemotherapy & & & 0.081 \\
\hline \multicolumn{4}{|l|}{ Regimen } \\
\hline Monochemotherapy & $35(22.1)$ & 313 & \\
\hline Doublet chemotherapy & $114(72.2)$ & 371 & \\
\hline Triple chemotherapy & $9(5.7)$ & 372 & \\
\hline Regimen containing oxaliplatin & $24(15.2)$ & 314 & 0.544 \\
\hline Regimen containing taxanes & $53(33.5)$ & 378 & \\
\hline Regimen containing Irinotecan and others & $50(31.6)$ & 364 & \\
\hline Others & $31(19.6)$ & 329 & \\
\hline Cycles (mean, 3 ; range, $1-12$ ) & & & $<0.001$ \\
\hline $1-2$ cycles & $65(41.2)$ & 292 & \\
\hline $3-4$ cycles & $50(31.6)$ & 378 & \\
\hline $5-6$ cycles & $28(17.7)$ & 478 & \\
\hline$>6$ cycles & $15(9.5)$ & 525 & \\
\hline Short-term efficacy & & & $<0.001$ \\
\hline $\mathrm{CR}(0)+\mathrm{PR}+\mathrm{SD}$ & $71(44.9)$ & 421 & \\
\hline $\mathrm{PD}$ & $87(55.1)$ & 301 & \\
\hline
\end{tabular}

mOS, median overall survival; CR, complete response; PR, partial response; SD, stable disease; PD, disease progression.

OS was 356 days. The further subgroup analyses yielded notable results. These disclosed that OS was significantly longer for the following two groups: Esophagogastric junction tumor site (Fig. 2A), and lymphatic metastasis (Fig. 2B). The result of the former subgroup is not consistent with previous reports, indicating that esophagogastric junction gastric cancer has poor prognosis. This inconsistency could be explained by the small number of cases, and the advances in nutritional treatment for patients with esophagogastric lesions that are currently being made. The results of the latter subgroup revealed that patients with lymphatic metastasis had improved survival rates compared with patients with peritoneal and hematogenous metastasis, and patients with a mixture of metastases had comparatively the worst survival. The histological factor appeared to be associated with OS, although the difference was not significant, which may be attributed to an insufficient number of cases in the present study. Other factors, including gender, age, and whether a radical operation was received, had no clear correlation with survival (Table I).

In addition, several of the treatment conditions, including treatment with $>2$ lines of chemotherapy (Fig. 2C), increasing 
Table III. Specific regimens used in first-line and second-line chemotherapy.

A, First-line chemotherapy

Chemotherapy regimen

Regimen group

Specific regimen

$\mathrm{n}(\%)$

Oxaliplatin-containing regimen $(\mathrm{n}=76)$

SOX

XELOX

FOLFOX

Taxane-containing regimen $(n=41)$

TX/DX

TP/DP

Irinotecan-containing regimen and others $(n=41)$

FOLFIRI

B, Second-line chemotherapy

Chemotherapy regimen

Regimen group

Specific regimen

n $(\%)$

Oxaliplatin-containing regimen $(n=24)$

SOX

XELOX

FOLFOX

Taxane-containing regimen $(\mathrm{n}=53)$

TX/DX

TP/DP

T, paclitaxel; D, docetaxel; SOX, S-1 plus oxaliplatin; XELOX, capecitabine plus oxaliplatin; TX, paclitaxel plus capecitabine; FOLFOX, folinic acid, fluorouracil and oxaliplatin; FOLFIRI, folinic acid, fluorouracil and irinotecan; DP, docetaxel and cisplatin; DS, docetaxel and S-1; DX, docetaxel plus capecitabine; DCF, docetaxel, cisplatin and fluorouracil; ECF, epirubicin, cisplatin and fluorouracil; TCF, paclitaxel, cisplatin and fluorouracil; FP, fluorouracil and cisplatin; XP, capecitabine and cisplatin; TS, paclitaxel plus S-1; DS, docetaxel plus S-1; IP, irinotecan plus cisplatin; IS, irinotecan plus S-1.

the number of cycles of chemotherapy (Fig. 2D and E), and good short-term efficacy of chemotherapy (Fig. 2F and G), led to markedly longer OS times. These observations highlighted that, if patients have a good tolerance to chemotherapy, they ought to be provided with more opportunities for chemotherapy. Furthermore, the administration of more drugs in combination may result in longer OS rates; although no significant differences were identified in the present study, this may have been due to the small number of cases involved. However, regimens with larger numbers of drugs may result in more severe side-effects. The National Comprehensive Cancer Network (NCCN) guidelines from 2015 (https://www.nccn.org/professionals/physician_gls/f_ guidelines.asp\#gastric) recommend that two-drug cytotoxic regimens are preferred as the first-line therapy, and the second-line therapy should be dependent on prior therapy and performance status. In the present study, two-drug cytotoxic regimens were routinely used $(84.8 \%$ in the first-line, and $72.2 \%$ in the second-line).

The most commonly used drugs in the treatment of AGC include oxaliplatin, taxanes, and irinotecan. Alternatively, fluoropyrimidine and cisplatin are often administered as basic drugs. In the first-line chemotherapy, three groups, including oxaliplatin-containing, taxane-containing and other regimens, had no clear correlation with survival. A randomized phase III study (AIO) demonstrated that irinotecan was able to prolong the OS in patients with AGC whose first-line treatment failed, compared with the BSC (13). Therefore, 

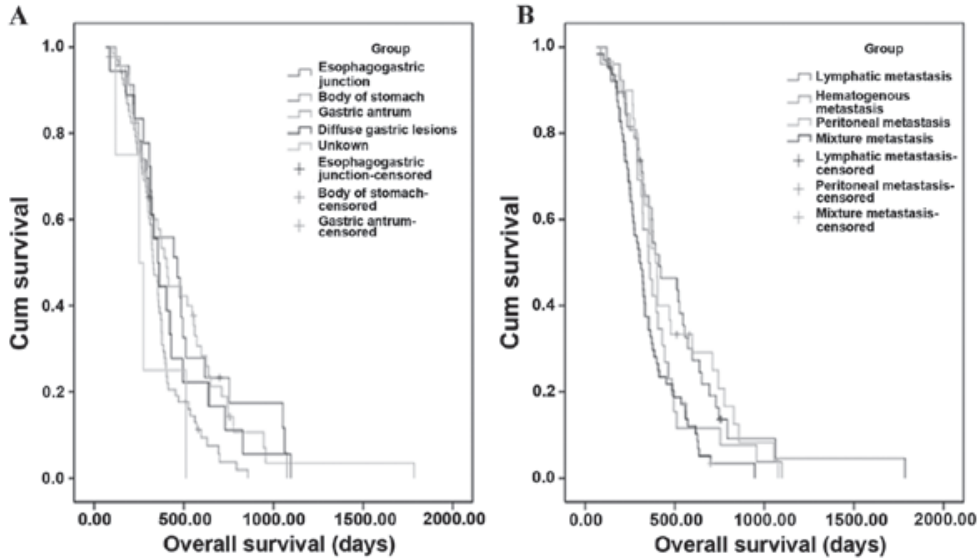

$\mathrm{C}$

D

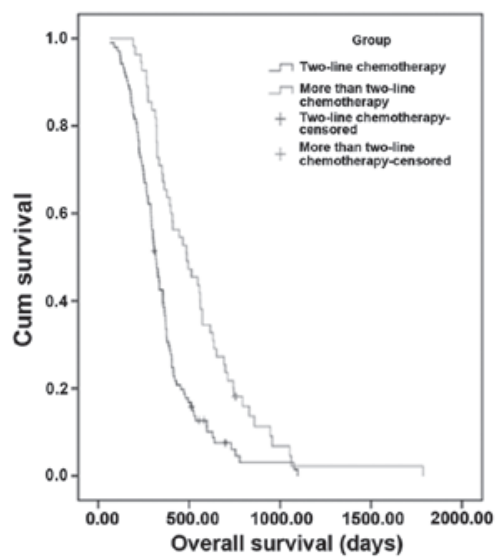

F

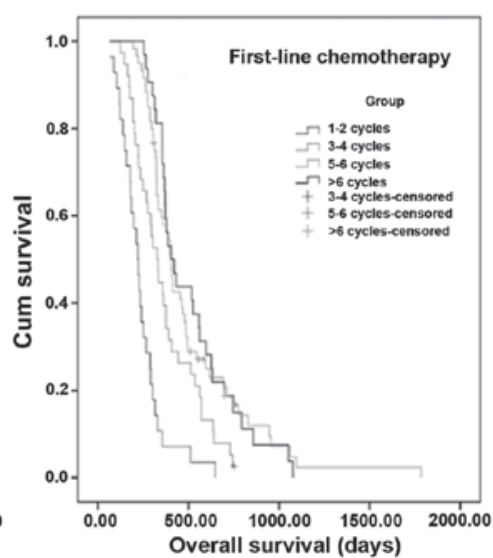

G
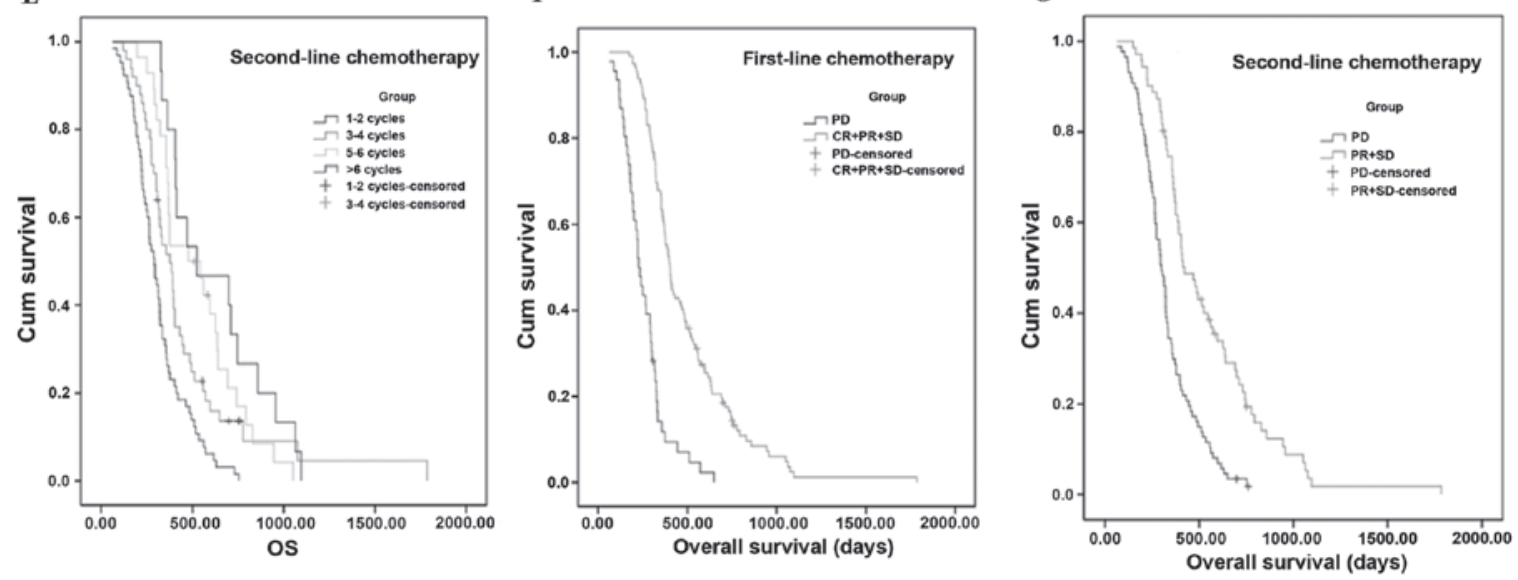

Figure 2. Subgroup analyses of OS. OS of the following subgroups were statistically significant: (A) Primary tumor site ( $\mathrm{P}=0.016)$; $(\mathrm{B})$ metastasis sites $(\mathrm{P}=0.005)$; (C) lines of chemotherapy; (D) cycles of first-line of chemotherapy $(\mathrm{P}<0.001)$; (E) Cycles of second-line of chemotherapy $(\mathrm{P}<0.001) ;(\mathrm{F})$ short-term efficacy of first-line of chemotherapy $(\mathrm{P}<0.001)$; and $(\mathrm{G})$ short-term efficacy of second-line of chemotherapy $(\mathrm{P}<0.001)$.

irinotecan-contained regimens were compared with other regimens as a second-line therapy. The median PFS was 90 days for the irinotecan-containing regimen group, 73 days for the oxaliplatin-containing regimen group, 105 days for the taxane-containing regimen group, and 94 days for the 'other regimens' group. No significant differences were identified among the different groups.

The current study had certain limitations. Conducting a retrospective analysis resulted in several differences between the numbers of patients in each of the groups. The toxicities were not recorded in detail. Nevertheless, the present study remains of certain consultative value for the performing of subsequent large-scale prospective clinical trials, and further validation of the conclusions reported in the present study are eagerly anticipated.

\section{Acknowledgements}

The present $\beta$ work was sponsored by the 1022 Talent Training Program of Zhejiang Cancer Hospital, the General Research Program of Medical Health in Zhejiang Province (grant nos. 2011KYA032, 2014KYB039 and 2016KYB036), the Scientific Research Fund Project of the Integrated Chinese and Western Medicine Institute in Zhejiang Province 
(no. 2014LYK021), the Science and Technology in Zhejiang Province Chinese Medicine Program (nos. 2012ZA101, 2015ZA148 and 2016ZA038), the Hangzhou City Science and Technology Project Planning Guide (Social Development; no. 20130733Q15), the Hangzhou City Health Science and Technology Project (grant no. 2013A43) and the Public Welfare Technology Application Studies Program of Zhejiang (grant no. 2015C33286).

\section{References}

1. Ferlay J, Soerjomataram I, Dikshit R, Eser S, Mathers C, Rebelo M, Parkin DM, Forman D and Bray F: Cancer incidence and mortality worldwide: Sources, methods and major patterns in GLOBOCAN 2012. Int J Cancer 136: E359-E386, 2015.

2. Tsai JY and Safran H: Status of treatment for advanced gastric carcinoma. Curr Oncol Rep 5: 210-218, 2003.

3. Power DG, Kelsen DP and Shah MA: Advanced gastric cancer slow but steady progress. Cancer Treat Rev 36: 384-392, 2010.

4. Wagner AD, Unverzagt S, Grothe W, Kleber G, Grothey A, Haerting $\mathrm{J}$ and Fleig WE: Chemotherapy for advanced gastric cancer. Cochrane Database Syst Rev: CD004064, 2010.

5. Sakuramoto S, Sasako M, Yamaguchi T, Kinoshita T, Fujii M, Nashimoto A, Furukawa H, Nakajima T, Ohashi Y, Imamura $\mathrm{H}$, et al: ACTS-GC Group: Adjuvant chemotherapy for gastric cancer with S-1, an oral fluoropyrimidine. N Engl J Med 357: 1810-1820, 2007.

6. Koizumi W, Narahara H, Hara T, Takagane A, Akiya T, Takagi M, Miyashita K, Nishizaki T, Kobayashi O, Takiyama W, et al: S-1 plus cisplatin versus $\mathrm{S}-1$ alone for first-line treatment of advanced gastric cancer (SPIRITS trial): A phase III trial. Lancet Oncol 9: 215-221, 2008.

7. Boku N, Yamamoto S, Fukuda H, Shirao K, Doi T, Sawaki A, Koizumi W, Saito H, Yamaguchi K, Takiuchi $\mathrm{H}$, et al: Fluorouracil versus combination of irinotecan plus cisplatin versus S-1 in metastatic gastric cancer: A randomised phase 3 study. Lancet Oncol 10: 1063-1069, 2009.

8. Sato Y, Takayama T, Sagawa T, Takahashi Y, Ohnuma H, Okubo S, Shintani N, Tanaka S, Kida M, Sato Y, et al: Phase II study of S-1, docetaxel and cisplatin combination chemotherapy in patients with unresectable metastatic gastric cancer. Cancer Chemother Pharmacol 66: 721-728, 2010.

9. Yamaguchi $\mathrm{H}$, Kitayama J, Ishigami $\mathrm{H}$, Emoto $\mathrm{S}$, Yamashita $\mathrm{H}$ and Watanabe T: A phase II trial of intravenous and intraperitoneal paclitaxel combined with S-1 for treatment of gastric cancer with macroscopic peritoneal metastasis. Cancer 119: 3354-3358, 2013.
10. Bang YJ, Kim YW, Yang HK, Chung HC, Park YK, Lee KH, Lee KW, Kim YH, Noh SI, Cho JY, et al: Adjuvant capecitabine and oxaliplatin for gastric cancer after D2 gastrectomy (CLASSIC): A phase III open-label randomised controlled trial. Lancet 379: 315-321, 2012.

11. Oki E, Emi Y, Kusumoto T, Sakaguchi Y, Yamamoto M, Sadanaga N, Shimokawa M, Yamanaka T, Saeki H, Morita M, et al: Phase II study of docetaxel and S-1 (DS) as neoadjuvant chemotherapy for clinical stage III resectable gastric cancer. Ann Surg Oncol 21: 2340-2346, 2014.

12. Bang YJ, Van Cutsem E, Feyereislova A, Chung HC, Shen L, Sawaki A, Lordick F, Ohtsu A, Omuro Y, Satoh T, et al: Trastuzumab in combination with chemotherapy versus chemotherapy alone for treatment of HER2-positive advanced gastric or gastro-oesophageal junction cancer (ToGA): A phase 3, open-label, randomized controlled trial. Lancet 376: 687-697, 2010.

13. Thuss-Patience PC, Kretzschmar A, Bichev D, Deist T, Hinke A, Breithaupt K, Dogan Y, Gebauer B, Schumacher G and Reichardt P: Survival advantage for irinotecan versus best supportive care as second-line chemotherapy in gastric cancer-a randomised phase III study of the Arbeitsgemeinschaft Internistische Onkologie (AIO). Eur J Cancer 47: 2306-2314, 2011

14. Kang JH, Lee SI, Lim do H, Park KW, Oh SY, Kwon HC, Hwang IG, Lee SC, Nam E, Shin DB, et al: Salvage chemotherapy for pretreated gastric cancer: A randomized phase III trial comparing chemotherapy plus best supportive care with best supportive care alone. J Clin Oncol 30: 1513-1518, 2012.

15. Kim HS, Kim HJ, Kim SY, Kim TY, Lee KW, Baek SK, Kim TY, Ryu MH, Nam BH and Zang DY: Second-line chemotherapy versus supportive cancer treatment in advanced gastric cancer: A meta-analysis. Ann Oncol 24: 2850-2854, 2013.

16. Shimizu T and Saijo N: Common toxicity criteria: Version 2.0 , an improved reference for grading the adverse reaction of cancer treatment. Nihon Rinsho 61: 937-942, 2003.

17. Eisenhauer EA, Therasse P, Bogaerts J, Schwartz LH, Sargent D, Ford R, Dancey J, Arbuck S, Gwyther S, Mooney M, et al: New response evaluation criteria in solid tumours: Revised RECIST guideline (version 1.1). Eur J Cancer 45: 228-247, 2009.

18. Glimelius B, Hoffman K, Haglund U, Nyrén O and Sjödén PO: Initial or delayed chemotherapy with best supportive care in advanced gastric cancer. Ann Oncol 5: 189-190, 1994.

19. Pyrhönen S, Kuitunen T, Nyandoto P and Kouri M: Randomised comparison of fluorouracil, epidoxorubicin and methotrexate (FEMTX) plus supportive care with supportive care alone in patients with non-resectable gastric cancer. Br J Cancer 71: 587-591, 1995. 